\title{
Galaxy interactions
}

\section{High density environments}

\author{
S. Alonso ${ }^{1,2}$, V. Mesa ${ }^{1,2}$, N. Padilla ${ }^{3}$, and D. G. Lambas ${ }^{1,4}$ \\ 1 Consejo Nacional de Investigaciones Científicas y Técnicas, Argentina \\ e-mail: salonso@icate-conicet.gob.ar \\ 2 ICATE, Universidad Nacional de San Juan, Argentina \\ 3 Pontificia Universidad Católica, Departamanto de Astronomía y Astrofísica \& Centro de Astroingeniería, Chile \\ ${ }^{4}$ IATE, OAC, Universidad Nacional de Córdoba, Laprida 854, X5000BGR, Córdoba, Argentina \\ Received 17 August 2011 / Accepted 9 November 2011
}

\begin{abstract}
Aims. To assess the role of dense environments in galaxy interactions, we present an analysis of close galaxy pairs in groups and clusters, obtained from the Sloan Digital Sky Survey Data Release 7 (SDSS-DR7).

Methods. We identified pairs that reside in groups by cross-correlating the total galaxy pair catalog with the SDSS-DR7 group catalog from Zapata et al. (2009, MNRAS, 394, 2229). We classify pair galaxies according to the intensity of interaction inferred from the morphological appearance of the optical images. We analyzed the effect of high-density environments on different classes of galaxygalaxy interactions and we studied the impact of the group global environment on pair galaxies.

Results. We find that galaxy pairs are more concentrated towards the group centers than the other group galaxy members, and disturbed pairs are more likely to contain the brightest galaxy in the groups. The color-magnitude relation of pair galaxies differs significantly from that of the control sample (constructed with galaxy group members without close companions), consisting in color tails with a clear excess of extremely blue and red galaxies for merging systems. In addition, pair galaxies show a significant excess of young stellar populations with respect to galaxies in the control sample; this finding suggests that, in dense environments, strong interactions have an important effect in modifying galaxy properties. We find that the fraction of star-forming galaxies decreases toward the group center; however, galaxy pairs have a more efficient star formation activity than galaxies without a close companion. We also find that pair galaxies tend to reside in groups in low density global environments with respect to galaxies of the corresponding control sample. Blue, young stellar population galaxies are also more likely to reside in groups within low density global environments. We find that this behavior is mainly driven by galaxy-galaxy interactions, which are the fundamental physical mechanisms driving this process.
\end{abstract}

Key words. galaxies: evolution - galaxies: interactions - galaxies: formation - galaxies: statistics - galaxies: star formation galaxies: groups: general

\section{Introduction}

Galaxy properties (morphology, color, star formation, gas content, etc.) have a strong dependence on the local environment in which galaxies reside. Several studies have found that galaxies in high-density local environment such as groups and clusters, have different properties from their isolated counterparts (e.g. Dressler 1980; Balogh et al. 2004; Baldry et al. 2006; Skibba \& Sheth 2009). Groups and clusters are dominated by spheroidal and gas poor galaxies, because when spiral galaxies traverse the dense environments of clusters, stripping removes their interstellar gas causing them to lose their ability to form new stars (e.g. Gunn \& Gott 1972; Dressler 1980).

In addition, observational and theoretical studies support the idea that galaxy properties also depend on the global environment. Some authors (e.g. Balogh et al. 2004; Ceccarelli et al. 2008; Park \& Choi 2009; Padilla et al. 2010) have indeed shown that the star formation rate (SFR) and colors depend on the large-scale structure. Using a semi-analytic model of galaxy formation, Gonzalez \& Padilla (2009) discovered evidence of a dependence on environment, namely that the fraction of red galaxies diminishes in equal local density environments, when farther away from clusters and closer to voids. Cooper et al. (2010) helped improve our understanding of these environmental dependences of galaxy properties by trying to relate them to the assembly history of the galaxy hosts. They found that for galaxies of equal age and mass, those characterized by younger ages (later assembly, and bluer colors) were preferentially located in low density environments. Lacerna \& Padilla (2011) explained this result by showing that the young stellar populations of galaxies in low density environments are the result of the uninterrupted growth of their host halo, which is not the case for galaxies of equal mass in higher density environments where the infall of material can be diverted to more massive neighbors.

On the other hand, observations show that mergers and galaxy interactions are powerful mechanisms that induce star formation (Kennicutt 1998), which may affect several properties of galaxies and their morphology. These effects strongly depend on the local environment and epoch in which galaxy interactions occur. Cosmological $N$-body simulations show that galaxies in close pairs are preferentially located in group environments (Barton et al. 2007). In the same direction, McIntosh et al. (2008) found that massive galaxy mergers are more likely to occur in large galaxy groups than in massive clusters. Heiderman et al. (2009) found a low merger rate that is responsible for only a small fraction of the total SFR density of the A901/902 superclusters. The predicted merger rates have 
largest uncertainties at the lowest masses and highest redshifts (Hopkins et al. 2009, 2010), and different assumptions about the modeling of galaxy mergers can also result in significant differences in the timings of mergers, with consequences for the formation and evolution of galaxies (De Lucia et al. 2010). Padilla et al. (2011) inferred the number of mergers during the evolution of early-type galaxies from $z=1$ to the present-day, finding that descendants of these galaxies at $z=0$ reside in environments of lower number density than their progenitors, implying that mergers must have decreased their number density before the present day.

Several previous observational studies have analyzed the role of the local density environment on galaxy interactions. Lambas et al. (2003) showed that pair galaxies (with projected distances of $r_{\mathrm{p}}<25 \mathrm{kpc} h^{-1}$ and relative radial velocities $\Delta V<$ $100 \mathrm{~km} \mathrm{~s}^{-1}$ ) in the field have a higher star formation activity than isolated galaxies in the same environment, with similar luminosity and redshift distributions. Alonso et al. (2004) performed a study of galaxy pairs in high-density regions corresponding to groups and clusters. The results of this study indicate that galaxy pairs in groups are systematically redder and have a lower star formation activity than other galaxy group members with no nearby companions, except for pairs with separations of $r_{\mathrm{p}}<15 \mathrm{kpc} h^{-1}$, which have a significantly higher activity of star formation. Alonso et al. (2006) obtained two galaxy pair catalogs from the 2-degree field Galaxy Redshift Survey (2dfGRS, Colless et al. 2001) and from the second data release of the Sloan Digital Sky Survey (SDSS-DR2, Abazajian et al. 2004), finding that the star formation birth rate parameter is a strong function of the local environment, $r_{\mathrm{p}}$ and $\Delta V$. Robaina et al. (2009) analyzed the enhancement in SFR as a function of projected galaxy separation in major mergers between massive galaxies. They found, on average, a mild enhancement in the SFR in pairs separated by projected distances $r_{\mathrm{p}}<40 \mathrm{kpch} h^{-1}$. Their work showed that galaxy interactions are more effective at triggering significant star formation activity in low and moderate density environments with respect to the control sample of galaxies without a close companion. Although close pairs have a larger fraction of actively star-forming galaxies and blue colors, there is a fraction of galaxy interactions that have redder colors than systems without a close companion.

Perez et al. (2009) found that in low and high local density environments, the colors and shapes of pairs of nearby galaxies are very similar to those of isolated galaxies; however, at intermediate densities they found significant differences, indicating that close pairs may have experienced a more rapid transition to the red sequence than isolated galaxies. In addition, they found that in intermediate-density environments galaxies are pre-processed efficiently by close encounters and mergers before entering groups and clusters. Using spectroscopic data from the PRIsm MUlti-object Survey (PRIMUS), Wong et al. (2011) studied isolated close galaxy pairs and compared these to a control sample in the redshift range $0.25<z<0.75$. They found that pair galaxies have bluer colors on average than isolated galaxies with both similar redshift and $r$-band luminosity distributions, which implies that they also have a higher SFR.

Ellison et al. (2010) used a sample of close galaxy pairs selected from the SDSS Data Release 4 (SDSS-DR4) to investigate the effect of the environment on interactions. They found that in high-density environments, mergers still occur but mainly without star formation; they also found that the bulge fraction is an increasing function of the nearest neighbor separation out to at least $400 \mathrm{kpc}^{-1}$. Previous studies (Alonso et al. 2007; Michel-Dansac et al. 2008; Lambas et al. 2012, hereafter L12) have also shown the importance of analyzing different classes of galaxy pair interactions. These studies found that merging pairs with strong signs of interaction have different properties (SFR, metallicity, color, etc.) from pairs that show no evidence of distorted morphologies.

Motivated by these findings, we analyze here the effect of high-density environments on different classes of galaxy-galaxy interactions and the role played by the global environment of the group on the properties of its number pairs. We study galaxy interactions within groups and clusters with the aim of assessing whether they play a significant role in modifying the properties of galaxies in pair systems, such as luminosities, colors, SFR, etc. For this purpose, using data from the seventh release of the SDSS (SDSS-DR7), we obtain a large sample of pair galaxies within groups and clusters (from Zapata el al. 2009) and we classify the interacting pairs according to their intensity of interaction as inferred from their morphological appearance in the optical images (Alonso et al. 2007, L11). We study the spatial distribution of galaxy pair colors and both stellar population and color-magnitude relations for different classes of galaxygalaxy interactions within groups/clusters, and compare them to those of group members without close galaxy companions, try to obtain additional clues about the interplay between the highdensity environment and merger properties. Finally, we analyze the effect of the group global environment, which is defined to be the distance to neighboring groups, to try and understand the influence of the large-scale structure on the galaxy formation processes.

This paper is structured as follows. Section 2 describes the procedure used to construct the catalog of group galaxy pairs from SDSS-DR7. Section 3 analyses the luminosity galaxy ranking and the pair distribution in groups/clusters. In Sect. 4, we discuss the distribution of galaxy pairs in groups and we study the colors and stellar population with respect to both group central distance and host group luminosity. In Sect. 5, we analyze the effect of the group global environment, and in Sect. 6 we summarize our main conclusions.

\section{Construction of a group galaxy pair catalog from SDSS-DR7}

The SDSS (York et al. 2000) is a photometric and spectroscopic survey that covers approximately one-quarter of the celestial sphere. The imaging portion of SDSS-DR7 (Abazajian et al. 2009) comprises 11663 square degrees of sky imaged in five wave-bands ( $u, g, r, i$, and $z$ ) containing photometric parameters of 357 million objects. The main galaxy sample is essentially a smaller, magnitude limited spectroscopic sample (Petrosian magnitude) with 929555 galaxies of $r_{\text {lim }}<17.77$. These galaxies span a redshift range $0<z<0.25$ with a median redshift of 0.1 (Strauss et al. 2002).

We calculated K-corrections using the publicly available code described in Blanton \& Roweis (2007) as a calibration for our K-corrected magnitudes. In our previous work (Alonso et al. 2007), we performed a suitable photometric analysis to assess the reliability of the SDSS magnitude deblending procedure in merging pairs, finding negligible systematic effects. As a spectral indicator of the mean stellar population age, we use the strength of the $4000 \AA$ break, $D_{n}(4000)$, defined as the ratio of the average flux densities in the narrow continuum bands (3850-3950 ̊ to 4000-4100 $\AA$, Balogh et al. 1999). This spectral discontinuity corresponds to an accumulation of spectral lines in a narrow region of the spectrum, an effect that is 
significant in the spectra of old stars. In the following analysis, we use the SFR normalized to the total mass in stars, $S F R / M^{*}$, taken from Brinchmann et al. (2004).

In our previous work (L11) on the SDSS-DR7, we compiled a galaxy pair catalog (GPC) selecting pairs with relative projected separations of $r_{\mathrm{p}}<25 \mathrm{kpc} \mathrm{h}^{-1}$, relative radial velocities of $\Delta V<350 \mathrm{~km} \mathrm{~s}^{-1}$, and redshifts $z<0.1$. In previous studies by the team, we found that these limits are adequate to define galaxy pairs with enhanced star formation activity (Lambas et al. 2003; Alonso et al. 2006). We excluded AGNs and removed false identifications (mostly parts of the same galaxy and objects with large magnitude uncertainties). With these restrictions, the pair catalog in the SDSS-DR7 comprises 1959 reliable close galaxy pairs with $z<0.1$. In the galaxy pair catalog obtained from the SDSS, the effects of incompleteness or aperture do not introduce significant biases (e.g see also Balogh et al. 2004; Alonso et al. 2006).

To analyze in detail the properties of galaxy interactions in high-density environments, we identified pairs that resides in groups by cross-correlating the total galaxy pair catalog with the SDSS-DR7 group catalog constructed by Zapata et al. (2009). These authors identified groups using a friends-of-friends algorithm with the same parameters corresponding to the values found by Merchan \& Zandivarez (2005) to produce a reasonably complete sample $(95 \%)$ with low contamination $(<8 \%)$.

The identification procedure consists of an improved version of the Huchra \& Geller (1982) friends-of-friends algorithm, with variable linking lengths of $D_{12}=D_{0} R$ and $V_{12}=V_{0} R$, in the direction perpendicular and parallel to the line-of-sight, respectively, where $D_{0}=0.24 h^{-1} \mathrm{Mpc}$ and $V_{0}=450 \mathrm{~km} \mathrm{~s}^{-1}$. The spatial scaling $R$ takes into account the variation in the space density of galaxies in a flux-limited catalog, and is calculated from the ratio of the density of galaxies brighter than the minimum required to enter the catalog at the mean distance of the galaxies being linked, to a characteristic survey depth (see Merchan \& Zandivarez 2005, for full details of the group catalog construction method).

In this work, we use the group luminosity as a proxy for the group mass, since the virial mass has been shown to be prone to large uncertainties below $M_{\text {vir }} \simeq 3 \times 10^{13} h^{-1} M_{\odot}$ (e.g., Padilla et al. 2004). Our estimator is also a proxy for the group total luminosity since our group luminosity consists of the addition of the luminosities of the four brightest galaxy members. The other important group property for this study is the group center, which we calculate by taking a luminosity weighted average of the positions of the member galaxies. The group catalog has 122962 galaxies within 12630 groups that have a minimum number of 4 members and $z<0.1$.

This catalog is constructed in a similar way to those of Berlind et al. (2006) and Yang et al. (2007), and is composed of groups of similar quality as is evident from comparing the completeness and contamination from these works and in Merchan \& Zandivarez (2005), while at the same time it gives us the added advantage of allowing us to perform the measurements of the group properties that we need.

As a result of this cross-correlation, we obtain a sample of 660 galaxy pairs in 615 groups, with $z<0.1$. We find 40 groups with 2 galaxy pairs, 3 groups with 3 galaxy pairs, and 2 groups with 4 galaxy pairs. Figure 1 shows an extraordinary and unusual example of a multiple galaxy interaction between five group members in the local Universe. The host galaxy group has 27 members with $M_{\text {Virial }}=2.12 \times 10^{13} M_{\odot}$.

Pair galaxies in the L11 catalog are classified as disturbed and non-disturbed pairs. The first class is further sub-classified

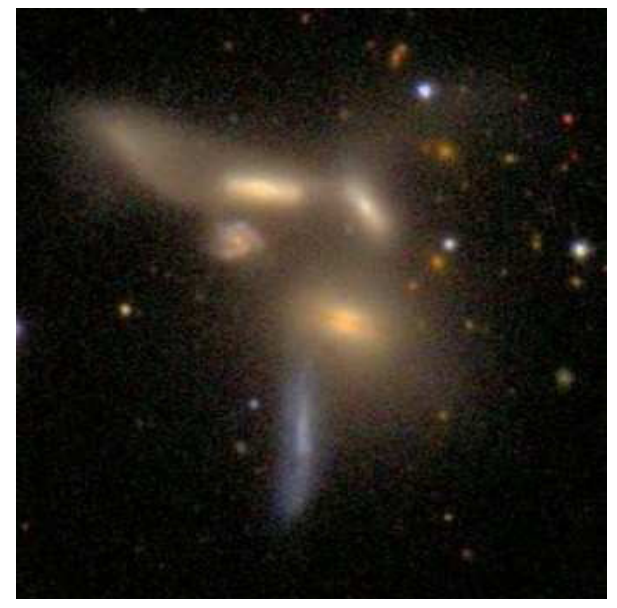

Fig. 1. Image of an extraordinary multiple interaction within a SDSS galaxy group. The host group has 27 members with $M_{\text {Virial }}=2.12 \times$ $10^{13} M_{\odot}$.

Table 1. Percentages of pairs classified as merging, tidal and nondisturbed, in SDSS groups.

\begin{tabular}{lcc}
\hline \hline Classification & Number of pairs & Percentages \\
\hline Merging & 58 & $8.8 \%$ \\
Tidal & 221 & $33.5 \%$ \\
Non-disturbed & 381 & $57.6 \%$ \\
\hline
\end{tabular}

into pairs that are merging $(M)$, which are those with evidence of an ongoing merging process, and tidal $(T)$ pairs, which display signs of tidal interactions but are not necessarily merging. Nondisturbed $(N)$ pairs show no evidence of distorted morphologies. For the group pair catalog, we calculate the percentage of these three categories defined above (see Table 1) and find that about $9 \%$ of galaxy pairs are classified as $M, 33 \%$ as $T$, and $58 \%$ as $N$.

Our samples can include spurious pairs. Mamon (1986, 1987 ) found that spurious pairs are expected to be more frequent in high-density regions. Our choices of limiting values for the projected separation and radial velocity help us to reduce this problem. We expect the $M$ and $T$ pairs to be mostly free of contamination owing to their distorted morphologies, but the $N$ pairs are particularly susceptible to this problem. To estimate the fraction of spurious pairs in our galaxy groups, we ran several Monte Carlo simulations of groups/clusters with more than 20 galaxies in $r_{\mathrm{p}}<1 h^{-1} \mathrm{Mpc}$. Our test shows that $98.2 \%$ of $M$ and $T$ pairs and $86.5 \%$ of $N$ pairs are real systems. From these estimates, we conclude that although spurious pairs are present in $N$ systems within dense regions, actual galaxy pairs clearly dominate the statistics.

We focus our attention on the effects of interactions in groups by comparing with a suitable control sample that differs from the group pair catalog only in that galaxies in groups in the control sample have no close companions. Using a Monte Carlo algorithm for each group galaxy pair, we select two members of the group catalog. In this paper, the control sample, therefore contains 1320 galaxies in groups and clusters that do not have a companion within $r_{\mathrm{p}}<100 \mathrm{~h}^{-1} \mathrm{kpc}$ and $\Delta V<350 \mathrm{~km} \mathrm{~s}^{-1}$. This comparison sample of galaxies in groups shares similar environmental conditions, ranges of redshift, $M_{r}$, and group luminosity distributions as the sample of galaxy pairs in groups (see Fig. 2). 

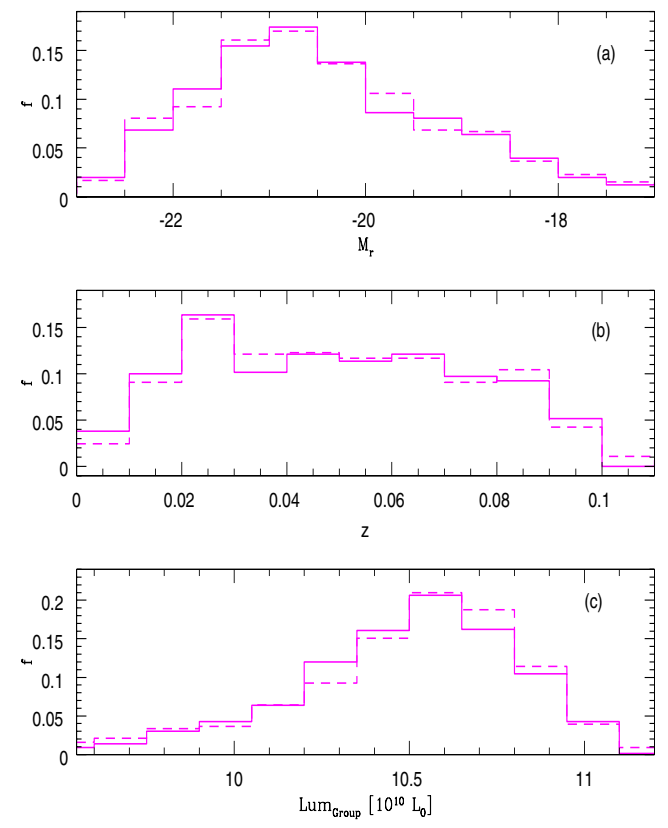

Fig. 2. Upper and medium panels a) and b) show the distributions of $M_{r}$ and $z$ in pair galaxies (solid lines) and in the control sample (dashed lines). The lower panel c) shows the group luminosity distribution for pair and control galaxies (solid and dashed lines, respectively).

\section{Luminosity galaxy ranking and pair distribution in groups}

The core of a cluster is a hostile, extreme environment that appears to promote the formation of less common galaxy types (Zwicky \& Humason 1960). The strongest evidence that mergers occur within clusters is the presence of a giant $\mathrm{cD}$ galaxy in the centers of clusters (Morgan et al. 1975), which have extraordinary luminosities and masses, well in excess of those of ordinary galaxies. The commonly accepted theory is that $\mathrm{cD}$ galaxies have cannibalized neighboring galaxies, accreting companions that stray into their domain. In addition, both observation and theory suggest that this process was more efficient in the past, when clusters were formed from smaller groups of galaxies with smaller relative velocities.

Von der Linden et al. (2007) found that the brightest group and cluster galaxies (BCGs) are more massive and have larger velocity dispersions than non-BCGs of the same stellar mass, suggesting that BCGs contain a larger fraction of dark matter. In addition, the peculiar velocity of the BCG is independent of cluster richness and only slightly dependent on the BautzMorgan type (Coziol et al. 2009). Zibetti et al. (2005) found that the surface brightness of the intracluster light (ICL) correlates with both the BCG luminosity and the cluster richness, while the fraction of total light in the ICL is almost independent of these quantities. In addition, it has been found that the ICL is a ubiquitous feature for clusters with a single, dominant BCG, which indicates that it dominates the combined luminosity of the BCG+ICL components (Gonzalez et al. 2005). Skibba et al. (2011) demonstrated that, frequently $(25 \%-40 \%)$ the brightest galaxies in groups or clusters are not the central galaxies of the systems.

In this section, we analyze the distribution of projected radial distance for pairs and all group galaxy members without near companions with respect to group centers. In all samples,

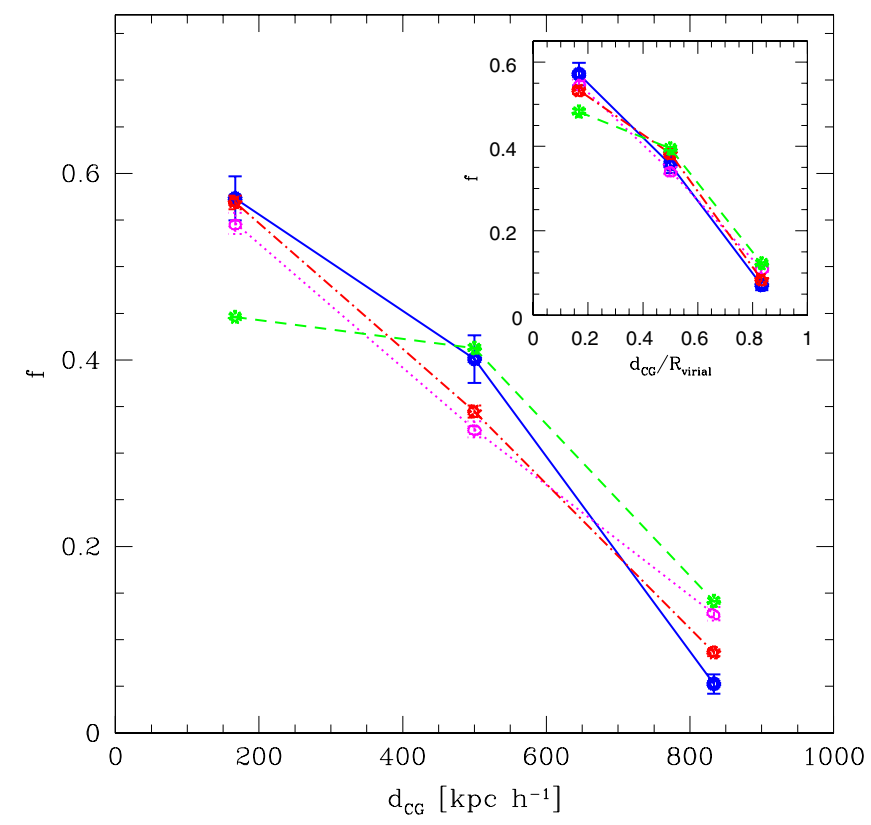

Fig. 3. Distribution of group-centric galaxy distances, $d_{\mathrm{CG}}$, for $M$ (solid lines), $T$ (dotted lines), and $N$ (dot-dashed lines) pairs. The dashed line represents all the group galaxy members without near companions. The small box corresponds to the distribution of the normalized groupcentric distance $d_{\mathrm{CG}} / R_{\mathrm{Virial}}$ in the same samples.

we have considered groups with a minimum number of ten members. The resulting distributions are shown in Fig. 3. It can be appreciated that galaxy pairs $(M, T$, and $N)$ are more concentrated towards the group center than the other group members. We also calculated the normalized group-centric distance, $d_{\mathrm{CG}} / R_{\text {Virial }}$, finding same tendencies (see the inset). Similar results were found by Ellison et al. (2010), who analyzed the dependence of interactions on cluster-centric distance, finding that for close pairs the fraction of asymmetric galaxies (associated with tidal disruptions) is highest in the cluster centers. Moreover, Lin et al. (2010) showed that the galaxy pair fraction increases with density.

We also study the luminosity galaxy ranking in pairs within groups (Fig. 4). The luminous galaxy in the group is ranked first, and so on for the second, third, etc., most luminous galaxies. It can be appreciated that disturbed pairs ( $M$ and $T)$ tend to contain the brightest group galaxy (45\% and $38 \%$ respectively, in all groups; $63 \%$ and $32 \%$ in groups with $\mathrm{Lum}_{\text {Group }}>10^{10.6} L_{\odot}$ ) with respect to non-disturbed pairs and other group galaxy members without near neighbors (see Table 2). These behaviors indicate that morphologically disturbed galaxy pairs are more concentrated in the central regions of the groups/clusters and contain preferentially the brightest object in the group. These results agree with the current paradigm of galaxy formation, which assumes that the structures form hierarchically, such that less massive halos merge to form more massive halos via, mostly, minor mergers. This would then imply that the central galaxy is generally the most luminous, most massive galaxy in a dark matter halo and resides at the center of the potential well of the halo, where it is also expected to cannibalize neighbor satellites.

\section{Colors and stellar population}

Different authors (Baldry et al. 2004; Balogh et al. 2004) showed that the distribution of galaxy colors could be closely described 


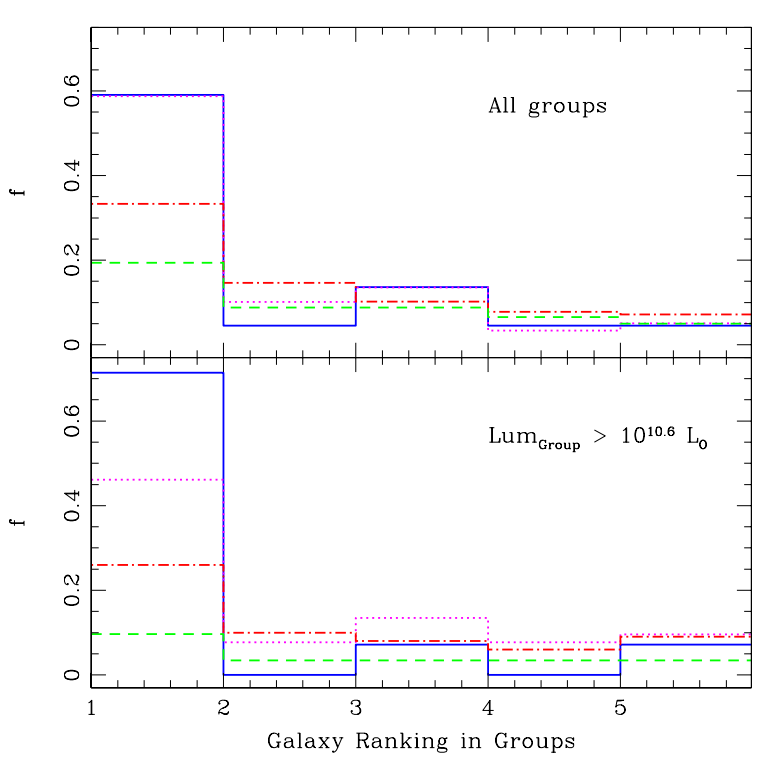

Fig. 4. Galaxy $r$-band luminosity ranking for different pairs classified as $M, T$ and $N$ (solid, dotted and dot-dashed lines, respectively) in all groups, with a minimum number of 4 members (upper panel) and in groups with $\operatorname{Lum}_{\text {Group }}>10^{10.6} L_{\odot}$ (lower panel). The dashed lines represent all the group galaxy members without near companions. The number 1 indicates the most luminous galaxy in the group, number 2 the second most luminous galaxy, and so on.

Table 2. Probability of different galaxy pair classes to be the brightest galaxy in the group/cluster.

\begin{tabular}{lcc}
\hline \hline $\begin{array}{l}\text { Pair } \\
\text { classification }\end{array}$ & \% in all groups & $\begin{array}{c}\text { \% in groups with } \\
\text { Lum }_{\text {Group }}>10^{10.6} L_{\odot}\end{array}$ \\
\hline$M$ & $45 \%$ & $63 \%$ \\
$T$ & $38 \%$ & $32 \%$ \\
$N$ & $24 \%$ & $14 \%$ \\
$G G M$ & $10 \%$ & $5 \%$ \\
\hline
\end{tabular}

Notes. GGM: group galaxy members without near companions.

by a double Gaussian with blue and red peaks over a wide range of absolute magnitudes and local environments.

Figure 5 shows color-magnitude diagrams for the different classes of pairs $M, T$, and $N$ (upper, middle, and lower panels, respectively) and for galaxies in the control sample (black contours in the three panels; hereafter we show the control sample results in all figures). As expected, galaxies in the control sample follow a bimodal distribution, with a high concentration in the red population. This behavior indicates that galaxies in high-density environments, such as groups and clusters, are preferentially red objects. Merging galaxies have a remarkably different color-magnitude relation from galaxies without near companions in the control sample where, for the same magnitude, they show a more scattered distribution, spreading toward blue and red populations. Tidal and non-disturbed pairs have a similar color-magnitude distribution to that of galaxies in the control sample (middle and lower panels, respectively).

To complement the previous analysis, Fig. 6 shows the stellar age indicator, $D_{n}(4000)$, as a function of $M_{r}$ for different pair classes ( $M, T$, and $N$; upper, middle, and lower panels, respectively) and the control sample (black solid contours). The group members without near neighbors in the control sample contain two distinct stellar populations, young and old. However, galaxy
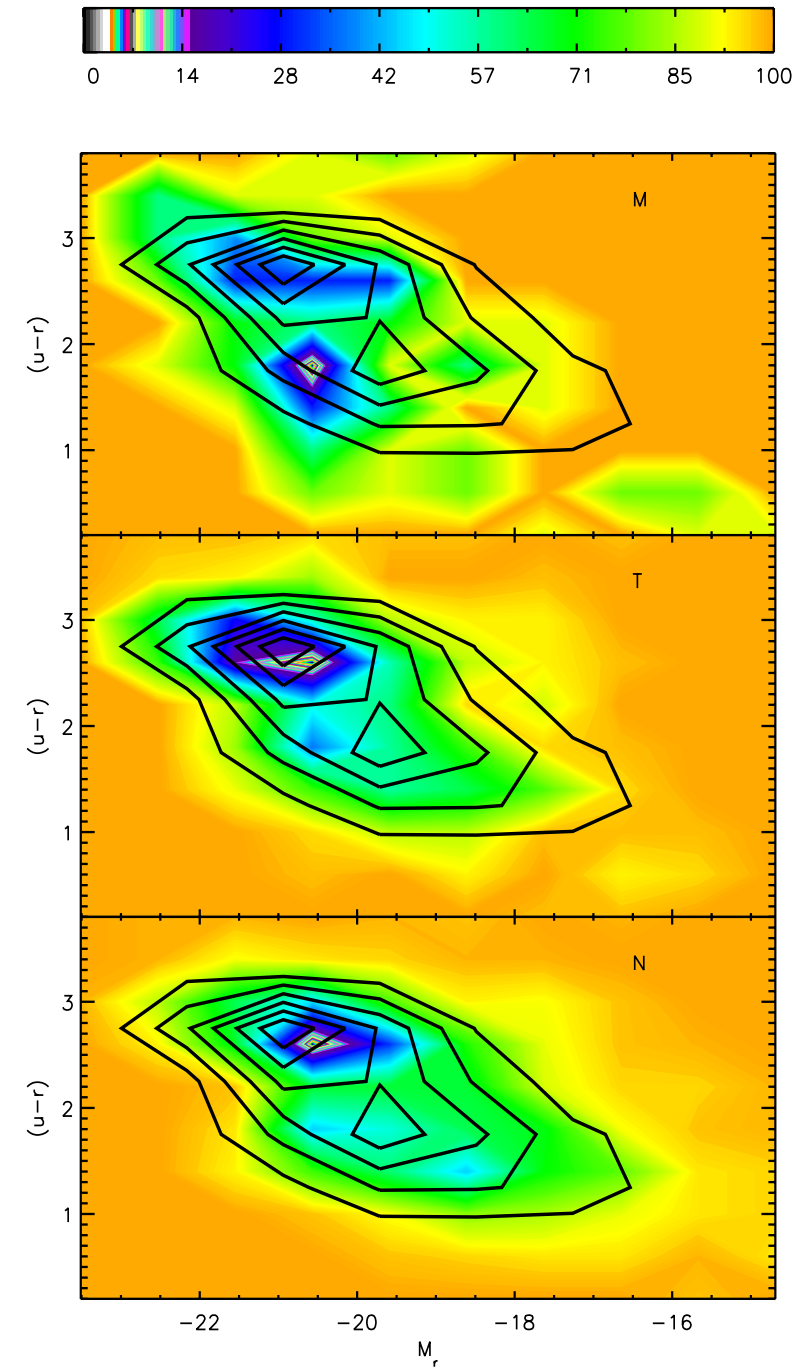

Fig. 5. Color-magnitude diagrams for merging, tidal, and non-disturbed pairs (upper, middle, and lower panels, respectively, in a color scale corresponding to the percentage of objects as shown in the key). For comparison, the black solid lines enclose $14 \%, 28 \%, 42 \%, 57 \%, 71 \%$, and $85 \%$ of the isolated galaxies in the control sample.

pairs clearly display an excess in the young stellar population sequence for the same galaxy magnitude, with respect to the control sample. This trend is more significant for merging systems.

In Fig. 7, we show the distributions of the $u-r$ color (left panels), $\log \left(S F R / M^{*}\right)$ (medium panels), and the stellar age indicator $D_{n}(4000)$ (right panels) for galaxy pairs and their isolated counterparts (galaxies in the control sample). We find that $T$ and $N$ systems (middle and lower panels, respectively) have color distributions that are more similar to those of the control sample than $M$ galaxy pairs. Interestingly, the color distribution of merging galaxy pairs and the control sample (upper panel) display significant differences, with pairs having larger fractions of red and blue members. This behavior could suggest that strong galaxy interactions are powerful mechanisms responsible for the fast and efficient transformation from blue to red colors in dense environments, compared to the less efficient interactions of isolated galaxies in the same density regions. In addition, the natural interpretation of the blue excess shown in pairs in groups is associated with the star formation activity triggered by galaxygalaxy interactions (e.g. Alonso et al. 2004; Perez et al. 2009). 


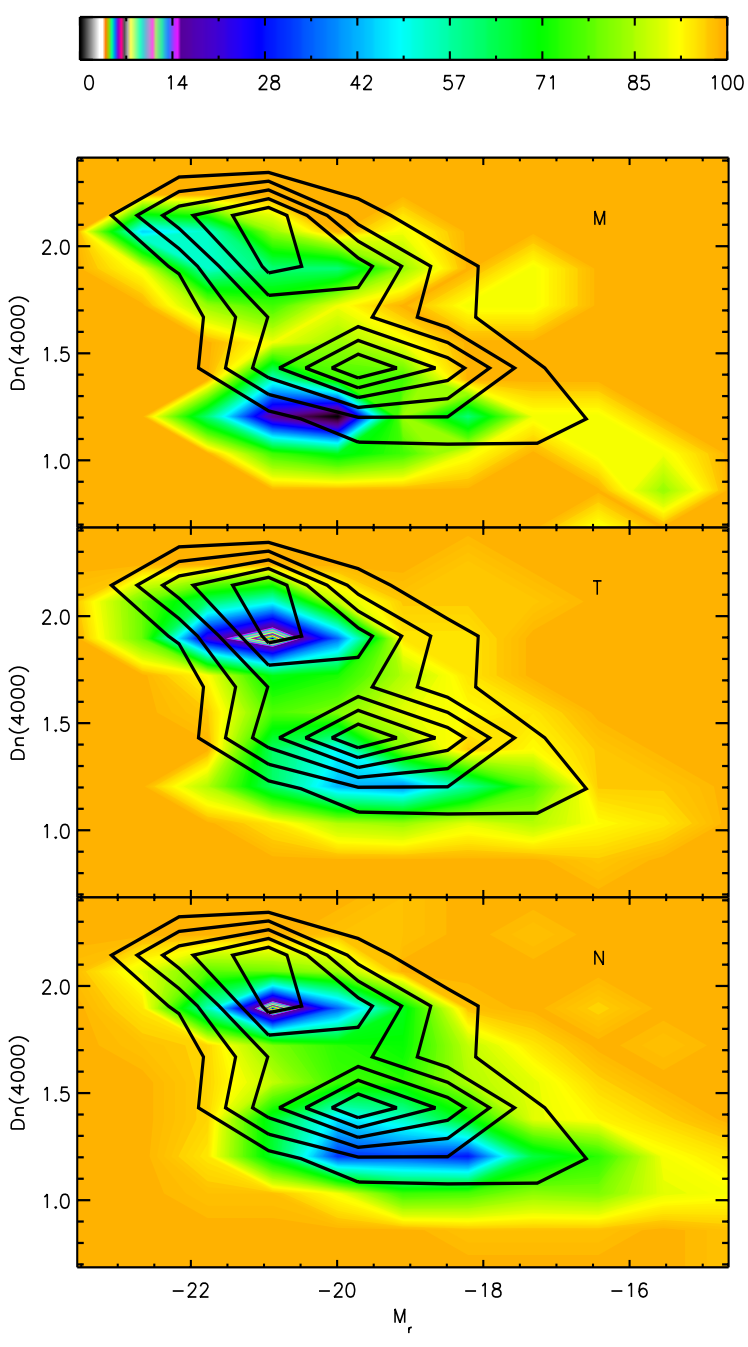

Fig. 6. Stellar age indicator, $D_{n}(4000)$ versus $M_{r}$ for merging, tidal, and non-disturbed pairs (upper, middle, and lower panels, respectively, in the color scale shown in the key). For comparison, the black solid lines enclose $14 \%, 28 \%, 42 \%, 57 \%, 71 \%$, and $85 \%$ of the isolated galaxies in the control sample.

The red excess in galaxy pairs has been reported by different authors (e.g. Alonso et al. 2006; Perez et al. 2009; Darg et al. 2010) but has no obvious interpretation. The red galaxy pairs might dusty, obscured star-forming systems. In this case, the slight spread at the red end in galaxy pairs might be due to higher extinction by the additional dust of the perturbing neighbor. To test this hypothesis, we studied color-color diagrams $((u-r)$. vs. $(i-z))$ for pairs $(M, T$ and $N)$ and isolated galaxies in the control sample. For the analysis, we selected edgeon $(b / a<0.4)$ and bright galaxies $\left(M_{r}<-20.0\right)$, at $z<0.1$, and for both samples we obtained diagrams with no appreciable differences. In another analysis, we divided the samples into ranges of $b / a$ and compared their color-color diagrams for each range. Unfortunately, with the available data it is impossible to confirm whether the dust is responsible for the red and old tails in disturbed pairs. Another possibility for the red excess is outlined by Patton et al. (2011). They computed galaxy colors using the Galaxy Image 2D (GIM2D, Simard et al. 2002), finding no significant excess $(<1 \%)$ of extremely red galaxies in close pairs. The authors suggest that this is due to higher quality photometry than other studies, given that they do find an excess of extremely red close pair members if they replace the GIM2D

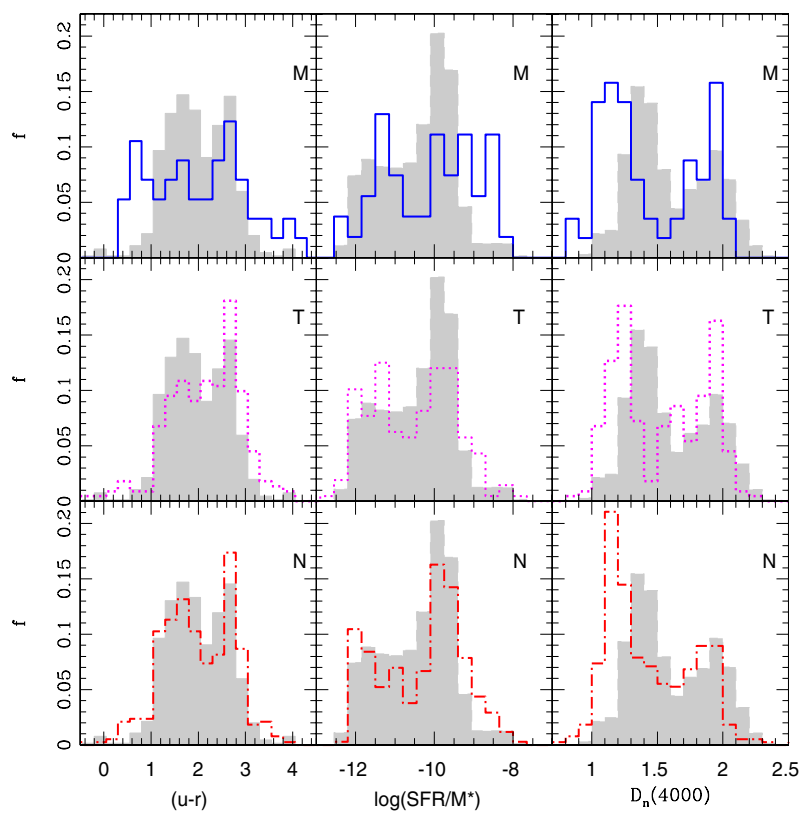

Fig. 7. Distributions of $u-r$ (left panels), $\log \left(S F R / M^{*}\right)$ (medium pan$e l s$ ), and $D_{n}(4000)$ (right panels) for different pair categories (solid lines): $M, T$, and $N$ (upper, middle, and lower panels, respectively) and the control sample (full surface).

colors with those from the SDSS database; they also find that the new photometry does not affect the fractions of extremely blue galaxies in pairs. On the other hand, different authors (e.g. Kauffmann et al. 2004; Baldry et al. 2006; Cooper et al. 2006; Skibba et al. 2009; Lin et al. 2010; Patton et al. 2011) show that paired galaxies are found in higher density environments than their control sample and that interacting galaxies are redder before the interaction starts, owing to the older stellar population present in higher density environments. However, pairs and isolated galaxies in our samples are selected from equal density environments i.e. groups and clusters. There is another plausible alternative explanation of this result. Red galaxies in pairs within groups and clusters have been stripped of their gas reservoir, consist primarily of old stellar populations, and hence the interactions are now unable to trigger further star formation activity. However, isolated galaxies in the control sample could include merger remnants as a consequence of the recently triggered star formation event, reflecting the bluer colors, as discussed in Perez et al. (2009).

The medium and right panels of Fig. 7 illustrate that there is a significantly higher star formation activity and younger stellar population in the three classes of interacting pairs than for isolated galaxies in the control sample. The correlation between colors, SFR, and stellar population indicates that the physical mechanism responsible for the color transformation also operates by changing, the star formation activity and stellar populations in galaxies.

\subsection{Dependence on the group-centric distance and the host group luminosity}

To help us understand the behavior of the colors and stellar populations in galaxy pairs within high-density environments, we measured the blue and young stellar population fractions with respect to the group-centric distance. In Fig. 8, we show the fraction of star-forming galaxies with $D_{n}(4000)<1.5$ (upper panel) and $u-r<2.0$ (lower panel) as a function of group-centric 


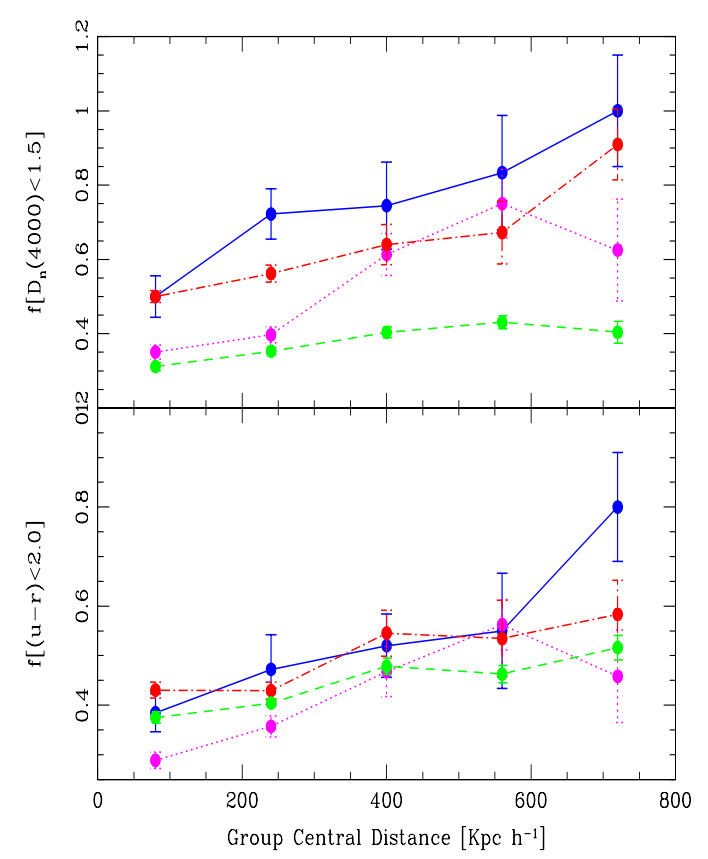

Fig. 8. Fraction of galaxies with $D_{n}(4000)<1.5$ (upper panel) and $(u-r)<2.0$ as a function of group-centric distance, for pairs classified as $M, T$, and $N$ (solid, dotted, and dot-dashed lines, respectively) and the control sample (dashed lines).

distance for both different classes of pairs and the control sample. As can be seen, the fraction of star-forming galaxies decreases toward the group center. Similarly, van den Bosch et al. (2008) found that redder satellite galaxies are somewhat more concentrated. In addition, Blanton \& Berlind (2007) showed that generally, the red galaxies are more clustered on small scales $\left(\approx 100-300 \mathrm{kpc}^{-1}\right)$. As is well-known, the central regions of the groups and clusters of galaxies are mainly populated by spherical objects, without gas, and truncated SFRs. It can also be appreciated that galaxy pairs have a higher star formation activity (low $D_{n}(4000)$ and blue colors) than galaxies without a close companion in the control sample. This tendency is more significant in merging pairs. This could indicate that merging pairs in high-density regions are still subject to a powerful mechanism that triggers star formation activity. On the other hand, we can discern a larger fraction of $T$ pairs with redder colors and older stellar populations. These results clearly show the longer timescale of morphological disturbances with respect to that of tidally induced star formation. Thus, these pairs may have old (reddened) stellar populations and still display strong signs of a past interaction. In addition, the strong tidal features can be associated with the disruption observed in some tidal interactions, which would lead to lower gas densities and therefore lower SFRs in these systems.

Figure 9 shows the fraction of star-forming and blue galaxies for which $D_{n}(4000)<1.5$ (upper panel) and $u-r<2.0$ (lower panel) for pairs as a function of the host group luminosity. We also show the results for the control sample (dashed lines). As can be seen, the fraction of star-forming galaxies decreases towards higher group luminosities. In addition, we can see that $M$ galaxy pairs have a systematically higher fraction of young stellar populations and bluer colors than both $T$ and $N$ pairs, and galaxies in the control sample. However, pairs displaying signs of tidal interactions have a lower star-formation activity than galaxies without close companions. Within the densest regions

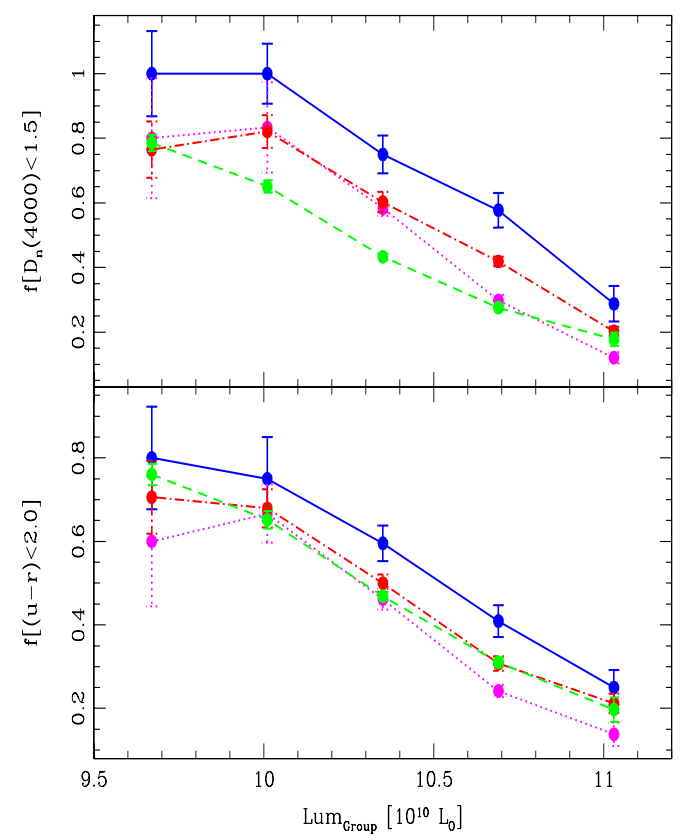

Fig. 9. Fraction of galaxies with $D_{n}(4000)<1.5$ (upper panel) and $(u-$ $r)<2.0$ as a function of the total group luminosity for pairs classified as $M, T$, and $N$ (solid, dotted, and dot-dashed lines, respectively) and the control sample (dashed lines).

(higher luminosity groups), the $M, T$, and $N$ pairs have similar SF activities to control galaxies, suggesting that in the densest environments the effects of pair interactions become overwhelmed by the intracluster processes.

\section{The effect of the group global environment}

We now analyse the influence of the group global environment on the properties of galaxy pairs in groups. For this purpose, we calculate the group global environment parameter, $\Sigma_{\mathrm{GR}}=$ $5 / \pi *\left(d_{\mathrm{GR}}^{5}\right)^{2}$, where $d_{\mathrm{GR}}^{5}$ is the distance to the fifth nearest group neighbor, which is calculated between the centers of groups. Group neighbors have been chosen to have luminosities above a certain threshold $\left(\right.$ Lum $\left._{\text {Group }}>10^{10.6} L_{\odot}\right)$ and a radial velocity difference that is smaller than $3000 \mathrm{~km} \mathrm{~s}^{-1}$.

The influence of the group global environment on the different classes of pairs can be seen in Fig. 10 in the histograms of $\log \left(\Sigma_{\mathrm{GR}}\right)$ for $M, T$, and $N$ pairs (upper, medium, and lower panels, respectively), and galaxies in the control sample (full surface). We also define the low and high-density environment classes by selecting two ranges of $\Sigma_{G R}$ values to have equal number of galaxies in these two classes in the control sample. This density threshold is $\log \left(\Sigma_{\mathrm{GR}}\right)=-1.2$ (represented by the dotted vertical line in Fig. 10). From this figure, it can be appreciated that galaxy pairs have an excess of low density group global environments with respect to the control sample. This behavior is more significant in $M$ pairs than in $T$ and $N$ systems $(65 \pm 7 \%$, $48 \pm 6 \%$, and $55 \pm 5 \%$, respectively).

We explored the stability of the results show in this figure, depending on the particular choice of global density estimator. We obtained $\Sigma_{\mathrm{GR}}$, using $d_{\mathrm{GR}}^{5}$, with two different group luminosity thresholds, Lum $_{\text {Group }}>10^{10.5} L_{\odot}$ and Lum $_{\text {Group }}>10^{10.3} L_{\odot}$. We also tested the results $\Sigma_{\mathrm{GR}}$ using the distance to the fourth group neighbor instead to the fifth group neighbor. Since the results of these tests are similar to within $10 \%$, we conclude that our 


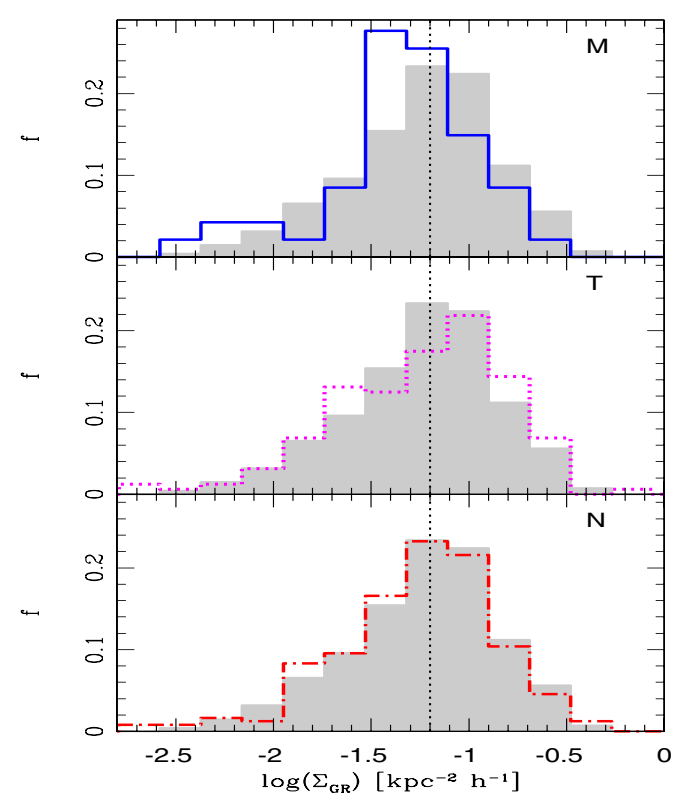

Fig. 10. Distribution of $\log \left(\Sigma_{\mathrm{GR}}\right)$ for different pair categories $M, T$, and $N$ (upper, middle, and lower panels, respectively) and the control sample (full surface).

analyses are independent of the particular definition of global group density environment.

To analyze the behavior of colors and stellar populations in pair systems and in galaxies in the control sample in groups and clusters with respect to the global density environment, we calculated the blue and young stellar population fractions $\left(u-r<2.0\right.$ and $D_{n}(4000)<1.5$, respectively) as a function of $\Sigma_{\mathrm{GR}}$. As can be seen (Fig. 11) for both samples (pair and control), the fraction of star-forming galaxies decreases towards higher density global environments. It can also be appreciated that galaxy pairs have a higher star-formation activity, which is particularly high in $M$ systems (lower $D_{n}(4000)$ and bluer colors), than galaxies without a close companion in the control sample. These results may indicate that young/old groups and clusters are associated with regions of low/high global overdensity. In this context, we note that galaxy pairs play a fundamental role in driving this relationship. In the same direction, Cooper et al. (2010), using samples drawn from the SDSS, studied the relationship between density and galaxy properties on the red sequence, finding that galaxies with older stellar populations tend to occupy regions of higher overdensity than galaxies of similar color and luminosity. In the study of Wang et al. (2007), they found that old, low mass halos at $z=0$ are associated with higher overdensities. Lacerna \& Padilla (2011) also found a population of halos that include nearby low mass halos in their original density peak, particularly for old objects. Our results confirm these previous findings for observational data.

\section{Summary and conclusions}

We have analyzed the properties of galaxy pairs in high-density environments corresponding to groups and clusters of galaxies. We now summarize the principal findings of our analysis and its main conclusions.

We have constructed the group galaxy pair catalog and classified the pair systems into three different categories, $M$, pairs with evidence of an ongoing merger process, $T$, pairs with signs of tidal interactions, and $N$, pairs showing no evidence of distorted morphologies. Using a Monte Carlo simulation, we

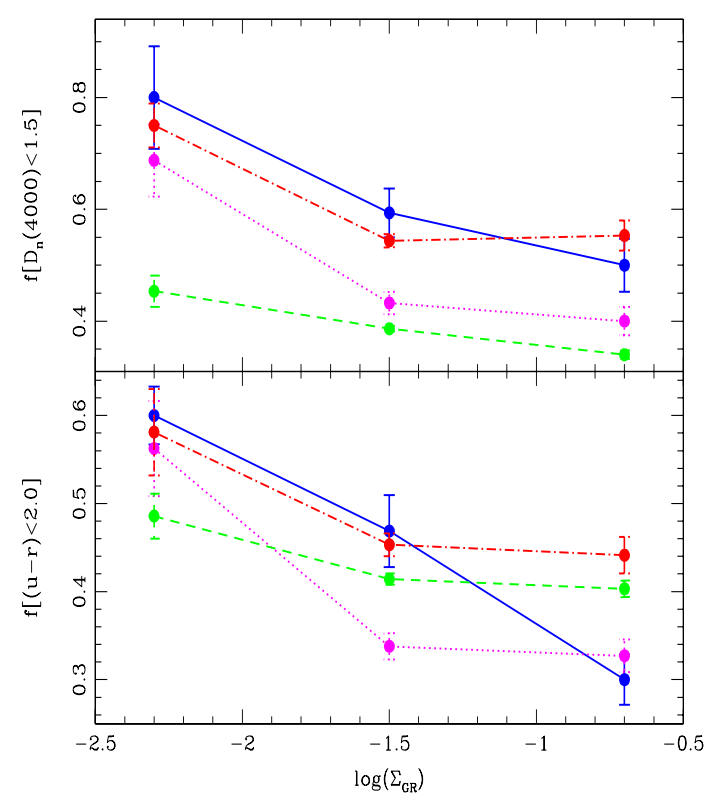

Fig. 11. Fraction of galaxies with $D_{n}(4000)<1.5$ (upper panel) and $(u-r)<2.0$ (lower panel) as a function of $\log \left(\Sigma_{\mathrm{GR}}\right)$, for pair galaxies classified as $M, T$, and $N$ (solid, dotted, and dot-dashed lines, respectively) and the control sample (dashed line).

estimated the contamination of spurious pairs in groups and clusters, finding that the statistics are clearly dominated by real pairs. We have found that about $9 \%$ of galaxy pairs are classified as $M$, $33 \%$ as $T$, and $58 \%$ as $N$. This implies that mergers are more likely to occur in less-dense groups, where the relative velocities of member galaxies are smaller than in richer groups/clusters ( $\approx 1000 \mathrm{~km} \mathrm{~s}^{-1}$; Toomre \& Toomre 1972; Dressler 1980).

We have found that galaxy pairs classified as $M, T$, and $N$ are more concentrated towards the group centers than the other group galaxy members. We have analyzed the luminosity galaxy ranking, finding that disturbed pairs ( $M$ and $T)$ have a preference to be the brightest galaxy in groups/clusters with respect to non-disturbed galaxy pairs and all group members.

We examined the color-magnitude relation for both different classes of pairs $(M, T$, and $N)$ and the control sample, in high-density environments. Galaxies in the control sample have a large red fraction, as expected for galaxies within groups and clusters. The $N$ pairs have a similar color-magnitude distribution. An interesting finding for $M$ galaxy pairs, is that their color distribution is significantly different in both color tails, with a clear excess of extremely blue and red objects. This tendency could indicate that merging systems within groups and clusters might have experienced a more rapid transition from blue to red colors, while isolated galaxies in the control sample and nondisturbed pairs undergo a more inefficient transformation.

In agreement with our color analysis, the distributions of SFR and stellar populations of $M, T$, and $N$ galaxy pairs have a clear excess of higher star formation activity and younger galaxies than galaxies in the control sample. This behavior is more noticeable in merging systems. The correlation found between bluer colors, higher star formation rate, and younger stellar populations in disturbed pairs suggests that strong interactions can significantly modify galaxy properties in dense environments.

We have studied the fraction of star-forming galaxies (those with blue colors and younger stellar populations) as a function of the group-centric distance in different pair classes and in the 
control sample. We have found that the number of star-forming galaxies decreases toward the group center as expected, but that galaxy pairs have a higher star formation activity than galaxies without a close companion within groups/clusters and that this is more significant in merging pairs. We speculate that this effect could indicate that in high-density regions, strong galaxy-galaxy interactions are a powerful mechanism that triggers star formation activity.

We have detected a significant correlation between the fraction of star-forming galaxies and the host group luminosity, where the number of star-forming objects increases towards low luminosity groups. In addition, we have found that $M$ galaxy pairs have a systematically larger fraction of younger stellar populations and bluer colors than the other pair categories and galaxies in the control sample.

We have found that galaxy pairs are more likely to reside within groups in low density global environments, than galaxies of the corresponding control sample. This behavior is more significant in $M$ pairs $(65 \pm 7 \%)$ than in both $T$ and $N$ systems $(48 \pm 6 \%$ and $55 \pm 5 \%$, respectively).

We have also analyzed the relation between star-forming galaxies and the group global environment for pairs and galaxies in the control sample. From this study, we conclude that, in particular, interacting galaxies with a dominant blue, young stellar population reside preferentially in groups within low density global environments. Since young groups and young clusters are associated with low density global environments, this is consistent with the idea that these structures are still assembling and being fed by a continuous supply of infalling material, which in turn promotes star formation activity. In this context, the history of galaxy interactions play a fundamental physical role in deciding the present star formation activity.

Acknowledgements. This work was partially supported by the Consejo Nacional de Investigaciones Científicas y Técnicas and the Secretaría de Ciencia y Técnica de la Universidad Nacional de San Juan. Nelson Padilla is supported by Centro de Astrofísica FONDAP, BASAL-CATA and Fondecyt Regular 1110328. Funding for the SDSS has been provided by the Alfred P. Sloan Foundation, the Participating Institutions, the National Science Foundation, the US Department of Energy, the National Aeronautics and Space Administration, the Japanese Monbukagakusho, the Max Planck Society, and the Higher Education Funding Council for England. The SDSS Web Site is http://www . sdss.org/. The SDSS is managed by the Astrophysical Research Consortium for the Participating Institutions. The Participating Institutions are the American Museum of Natural History, Astrophysical Institute Potsdam, University of Basel, University of Cambridge, Case Western Reserve University, University of Chicago, Drexel University, Fermilab, the Institute for Advanced Study, the Japan Participation Group, Johns Hopkins University, the Joint Institute for Nuclear Astrophysics, the Kavli Institute for Particle Astrophysics and Cosmology, the Korean Scientist Group, the Chinese Academy of Sciences (LAMOST), Los Alamos National Laboratory, the Max-Planck-Institute for Astronomy (MPIA), the Max-Planck-Institute for Astrophysics (MPA), New Mexico State University, Ohio State University, University of Pittsburgh, University of Portsmouth, Princeton University, the United States Naval Observatory, and the University of Washington. We thank Laura Balmaceda for her useful advice in IDL codes. We thank Ramin Skibba for a detail revision that helped to improve this paper.

\section{References}

Abazajian, K., Adelman, J., Agueros, M., et al. 2004, AJ, 128, 502

Abazajian, K. N., Adelman-McCarthy, J. K., Agüeros, M. A., et al. 2009, ApJS, 182,543

Alonso, M. S., Tissera, P. B., Coldwell, G., \& Lambas, D. G. 2004, MNRAS, 352,1088

Alonso, M. S., Lambas, D. G., Tissera, P., \& Coldwell, G. 2006, MNRAS, 367, 1029
Alonso, M. S., Lambas, D. G., Tissera, P., \& Coldwell, G. 2007, MNRAS, 375, 1017

Baldry, I. K., Glazebrook, K., Brinkmann, J., et al. 2004, ApJ, 600, 681

Baldry, I. K., Balogh, M. L., Bower, R. G., et al. 2006, MNRAS, 373, 469

Balogh, M., \& Berlind, A. 2007, ApJ, 664, 791

Balogh, M., Morris S. L., Yee, H. K. C., Carlberg, R. G., \& Ellingson, E. 1999, ApJ, 527, 54

Balogh, M., Eke, V., Miller, C., et al. 2004, MNRAS, 348, 1355

Barton, E. J., Arnold, J. A., Zentner, A. R., et al. 2007, ApJ, 671, 1538

Berlind, A. A., Frieman, J., Weinberg, D. H., et al. 2006, ApJS, 167, 1

Blanton, M., \& Roweis, S. 2007, AJ, 133, 734

Brinchmann, J., Charlot, S., White, S. D. M., et al. 2004, MNRAS, 351, 1151

Ceccarelli, L., Padilla, N., \& Lambas, D. G. 2008, MNRAS, 390, L9

Colless, M. M., Dalton, G., Maddox, S., et al. (the 2dFGRS Team) 2001, MNRAS, 328, 1039

Cooper, M. C., Newman, J. A., Croton, D. J., et al. 2006, MNRAS, 370, 198

Cooper, M. C., Gallazzi, A., Newman, J. A., \& Yan, R. 2010, MNRAS, 402, 1942

Coziol, R., Andernach, H., Caretta, C. A., Alamo-Martínez, K. A., \& Tago, E. 2009, AJ, 137, 4795

Darg, D. W., Kaviraj, S., Lintott, C. J., et al. 2010, MNRAS, 401, 1552

De Lucia, G., Boylan-Kolchin, M., Benson, A. J., et al. 2010, MNRAS, 406, 1533

Dressler, A. 1980, ApJ, 236, 351

Ellison, S. L., Patton, D. R., Simard, L., et al. 2010, MNRAS, 407, 1514

González, R. E., \& Padilla, N. D. 2009, MNRAS, 397, 1498

González, R. E., Padilla, N. D., Galaz, G., \& Infante, L. 2005, MNRAS, 363, $1008 \mathrm{G}$

Gunn, J. E., \& Gott, J. R. 1972, ApJ, 176, 1

Heiderman, A., Jogee, S., Marinova, I., et al. 2009, ASPC, 419, 257

Hopkins, P. F., Somerville, R. S., Cox, T. J., et al. 2009, MNRAS, 397, 802

Hopkins, P. F., Croton, D., Bundy, K., et al. 2010, ApJ, 724, 915

Huchra J. P., \& Geller M. J. 1982, ApJ, 257, 423

Kennicutt, R. 1998, ARA\&A, 36, 189

Kauffmann, G., White, S. D. M., Heckman, T. M., et al. 2004, MNRAS, 353, $713 \mathrm{~K}$

Lacerna, I., \& Padilla, N. 2011, MNRAS, 412, 1283

Lambas, D. G., Tissera, P. B., Alonso, M. S., \& Coldwell, G. 2003, MNRAS, 346,1189

Lambas, D. G., Alonso, M. S., Mesa, V., \& O’Mill, A. L. 2012, A\&A, 539, A45

Lin, L., Cooper, M. C., Jian, H.-Y., et al. 2010, ApJ, 718, 1158

Mamon, G. A. 1986, ApJ, 307, 426

Mamon, G. A. 1987, ApJ, 321, 622

McIntosh, D., Guo, Y., Hertzberg, J., et al. 2008, MNRAS, 388, 1537

Merchán, M., \& Zandivarez, A. 2005, ApJ, 630, 759

Michel-Dansac, L., Lambas, D. G., Alonso, M. S., \& Tissera, P. 2008, MNRAS, 386, L82

Morgan, W. W., Kayser, S., \& White, R. A. 1975, ApJ, 199, 545

Padilla, N., Baugh, C. M., Eke, V. R., et al. (The 2dFGRS Team) 2004, MNRAS, 352,211

Padilla, N., Lambas, D. G., \& González R. 2010, MNRAS, 409, 936

Padilla, N., Christlein, D., Gawiser, E., Marchesini, D. et al. 2011, A\&A, 531, A142

Park, C., \& Choi, Y.-Y. 2009, ApJ, 691, 1828

Patton, D. R., Ellison, S. L., Simard, L., McConnachie, A. W., \& Mendel J. T. 2011, MNRAS, 412, 591

Pérez, J., Tissera, P., Padilla, N., Alonso, M. S., \& Lambas, D. G. 2009, MNRAS, 399,1157

Robaina, A., Bell, E. F., Skelton, R. E., et al. 2009, ApJ, 704, 324

Simard, L., Willmer, C. N. A., Vogt, N. P., et al. 2002, ApJS, 142, 1

Skibba, R. A., \& Sheth, R. K. 2009, MNRAS, 392, 1080

Skibba, R. A., van den Bosch, F. C., Yang, X., et al. 2011, MNRAS, 410, 417

Strauss, M. A., Weinberg, D. H., Lupton, R. H., et al. 2002, AJ, 124, 1810

Toomre, A., \& Toomre J. 1972, ApJ, 178, 623

van den Bosch, Frank, C., et al. 2008, unpublished [arXiv:0805.0002V]

von der Linden, A., Best, P. N., Kauffmann, G., White, S. D. M., et al. 2007, MNRAS, 379, 867

Wang, H. Y., Mo, H. J., \& Jing, Y. P. 2007, MNRAS, 375, 633

Wong, K., Blanton, M. R., Burles, S. M., et al. 2011, ApJ, 728, 119

Yang, X., Mo, H. J., van den Bosch, F., et al. 2007, ApJ, 671, 153

York, D. G., Adelman, J., Anderson, J. E. Jr., et al. 2000, AJ, 120, 1579

Zapata, T., Pérez, J., Padilla, N., \& Tissera, P. 2009, MNRAS, 394, 2229

Zibetti, S., White, S. D. M., Schneider, D. P., \& Brinkmann, J. 2005, MNRAS 358,949

Zwicky, F., \& Humason, M. L. 1960, ApJ, 132, $627 Z$ 